\title{
Zerumbone decreases BACH1 levels by upregulating miR- 708 to inhibit breast cancer cell proliferation and invasion
}

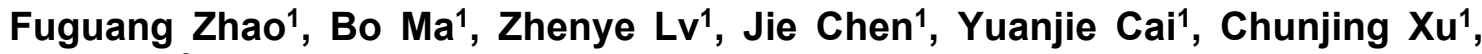 \\ Yong Cai ${ }^{2 *}$ \\ ${ }^{1}$ Department of Breast Surgery, Zhejiang Hospital, Hangzhou City, Zhejiang Province 310030, ${ }^{2}$ The First Department of Breast \\ Surgery, Jilin Cancer Hospital, Changchun City, Jilin Province 130012, China
}

*For correspondence: Email: ANBU61kl@163.com; Tel: +86-431-85879136

\begin{abstract}
Purpose: To investigate the potential mechanism by which zerumbone suppresses breast cancer (BC) cells.

Methods: Cell viability and Transwell assays were performed to assess the effect of zerumbone on BC cell growth. The downstream target of zerumbone was determined using quantitative polymerase chain reaction assays and immunoblotting. Cell viability assays and immunoblotting were conducted to detect if zerumbone had any effect on BACH1 (BTB domain and CNC homolog 1) expression.

Results: Zerumbone suppressed the proliferation, migration, and invasion of $B C$ cells. It also upregulated the expression of microRNA (miR)-708 and, hence, suppressed BACH1 expression. Furthermore, zerumbone suppressed the proliferation and invasion of BC cells by promoting miR-708 expression and suppressing $\mathrm{BACH} 1$.

Conclusion: The findings help clarify the anti-tumor mechanism of zerumbone and provide theoretical and therapeutic bases for the anti-tumor effects of Chinese herbal medicine.
\end{abstract}

Keywords: Breast cancer, Zerumbone, Cell invasion, MiR-708, BACH1

\begin{abstract}
This is an Open Access article that uses a fund-ing model which does not charge readers or their institutions for access and distributed under the terms of the Creative Commons Attribution License (http://creativecommons.org/licenses/by/4.0) and the Budapest Open Access Initiative (http://www.budapestopenaccessinitiative.org/read), which permit unrestricted use, distribution, and
\end{abstract} reproduction in any medium, provided the original work is properly credited.

Tropical Journal of Pharmaceutical Research is indexed by Science Citation Index (SciSearch), Scopus, International Pharmaceutical Abstract, Chemical Abstracts, Embase, Index Copernicus, EBSCO, African Index Medicus, JournalSeek, Journal Citation Reports/Science Edition, Directory of Open Access Journals (DOAJ), African Journal Online, Bioline International, Open-J-Gate and Pharmacy Abstracts

\section{INTRODUCTION}

Breast cancer (BC) is the most common gynecological cancer, with the highest incidence among female malignant tumors [1]. Advanced $\mathrm{BC}$ has a poor prognosis due to its high metastasis rate and heterogeneity [2]. The standard treatments for advanced BC include surgery and chemoradiotherapy [3]. Surgical resection often results in $\mathrm{BC}$ recurrence, while chemoradiotherapy is associated with significant side effects, and the low concentration of chemoradiotherapy drugs at the tumor site limits its effects on tumor tissues [4]. Common antitumor drugs for BC have poor penetration and can induce drug resistance, limiting their efficacy [5]. Therefore, it is necessary to develop new treatments to improve the prognosis of patients with advanced BC.

Zerumbone is a type of double half mushroom compound extracted from ginger rhizomes demonstrated to have a variety of biological effects such as anti-inflammation and 
ameliorating behavioral impairments [6]. Importantly, anti-tumor effects of zerumbone have been described in recent decades [7]. A previous study reported that zerumbone affected cell proliferation and induced cell cycle arrest in human laryngeal carcinoma HEP-2 cells [8]. It was shown to inhibit invasion by human nonsmall cell lung cancer (NSCLC) cells via the FAK/AKT/ROCK pathway [9]. Zerumbone also enhanced apoptosis in human colon cancer cells by inducing the death receptor, and it suppressed angiogenesis in pancreatic cancer by blocking nuclear factor (NF)-KB [10]. In BC, zerumbone was found to suppress $B C$ cell invasion and induce apoptosis [11]. However, the potential mechanism remains unclear.

Zerumbone exerts biological activity by affecting microRNAs (miRNAs) [12]. For example, it attenuated obesity induced by a high-fat diet by regulating miR-146B levels and sirtuin 1mediated fat production [13]. One recent study demonstrated decreased miR-708 expression in $B C$, and up-regulation of miR-708 suppressed tumor growth and drug resistance [14]. Here, we performed experiments to better understand the regulatory effects of zerumbone on BC progression.

\section{EXPERIMENTAL}

\section{Cell culture}

The BC cell line MDA-MB-231 was obtained from the American Type Culture Collection (ATCC, USA) and incubated in Dulbecco's modified Eagle's medium (DMEM) supplemented with 10 $\%$ fetal bovine serum (Invitrogen, USA) at $37{ }^{\circ} \mathrm{C}$ with $5 \% \mathrm{CO}_{2}$.

\section{Cell viability assay}

Cells were incubated in 96-well plates at a density of $2 \times 10^{3}$ cells in $100 \mu \mathrm{L}$ medium per well for $24 \mathrm{~h}$ before use in experiments to allow total adherence. Cell viability was assessed using MTT (3-(4,5-dimethylthiazol-2-yl)-2,5diphenyltetrazolium bromide) assays according to the manufacturer's instructions.

\section{Colony formation assay}

BC cells $\left(1 \times 10^{3}\right)$ were seeded into 6 -well culture plates and incubated for 2 weeks. For visualization, cells were fixed with paraformaldehyde (PFA) for $10 \mathrm{~min}$, and then stained with crystal violet staining solution and photographed. The numbers of colonies were counted manually.

\section{Cell migration and invasion assays}

Wound healing assays were used to determine cell migration. The cell layer was wounded by scraping with a $200-\mu \mathrm{L}$ tip, followed by washing. Subsequently, serum-free culture medium was added to stimulate wound healing. Photographs were taken at 0 and $24 \mathrm{~h}$ to evaluate cell migration.

Transwell chambers (8- $\mu \mathrm{m}$ pore size; Corning, USA) with $20 \%$ Matrigel in the upper chamber were filled with BC cells (Becton Dickinson, USA). Complete medium was added to the bottom chamber. After $48 \mathrm{~h}$ of incubation, cells in the upper chamber were induced to migrate toward the bottom chamber. Then the upper chamber medium and the lower chamber cells were fixed in PFA, stained with $0.1 \%$ crystal violet, and counted under a microscope.

\section{Quantitative polymerase chain reaction (PCR)}

Total RNA was extracted from cells by TRIzol reagent (Invitrogen, USA) and reversetranscribed with a Transcriptor First Strand cDNA Synthesis Kit (Takara, Japan). A SYBR Ex Taq kit (Takara) was used to amplify indicated genes.

The expression levels of related genes were normalized to glyceraldehyde 3-phosphate dehydrogenase (GAPDH). For miR-708 quantitative PCR (qPCR), we used a TaqMan microRNA RT kit (Applied Biosystems, USA). The primers used in this assay were listed as follows:

BACH1 (BTB domain and CNC homolog 1): forward: 5'- ATTCATGCTTCTGTTCAGCCAA-3', Reverse: 5'- GGCACTGAGAAGCAGGATCTTT3'; GAPDH: forward: 5'-CTCTGCTCCTCCTG TTCGAC-3', Reverse: 5'-ACCAAATCCGTTGA CTCCGA-3'.

\section{Luciferase reporter assay}

BACH1 primers (Forward: 5'-CAGACTAGTA AGCCAATGGAACCCTTGATT-3' and Reverse: 5'-CAGAAGCTTGCCTTGAAACATTTTCTTAGA

A-3') were used for amplification. BC cells were seeded in 12-well plates 1 day before transfection with pGL3-wt-Bach1-UTR, pGL3mu-Bach1-UTR, miR-708 mimic, or negative control mimics using Lipofectamine 2000 (Invitrogen). At $48 \mathrm{~h}$ after transfection, the cells were collected for relative luciferase activity measurement using Dual Luciferase Assays (Cat\#: E1910, Promega, USA). 


\section{Immunoblotting}

Cells were harvested and lysed for protein extraction. Denatured protein samples were separated with sodium dodecyl sulfatepolyacrylamide gel electrophoresis (SDS-PAGE) and then transferred to polyvinylidene fluoride membranes (Millipore Corporation, USA). Antibodies used for immunoblot analysis were against BACH1 (1:200 dilution, sc-14700, Santa Cruz Biotechnology, USA), matrix metalloproteinase (MMP)2 and -9 (1:1500 dilution, Santa Cruz Biotechnology), and GAPDH antibody (1:3000 dilution, sc-32233, Santa Cruz Biotechnology) was used as loading control. All protein bands were detected using an enhanced chemiluminescent substrate (Cell Signaling Technology, USA).

\section{Statistics}

GraphPad 5.0 software was used for all statistical analyses. Data are presented as mean \pm SEM. Student' t-tests was performed to compare groups, and $p<0.05$ was considered significant.

\section{RESULTS}

Zerumbone inhibits BC cell proliferation, migration, and invasion

Viability assay was performed using MDA-MB231 cells treated with different doses of zerumbone to investigate its effects on BC cell proliferation. Cell viability was reduced after exposure to higher concentrations of zerumbone (Figure $1 \mathrm{~A}$ ). Next, Transwell assays were performed. Zerumbone significantly reduced both $\mathrm{BC}$ cell migration and invasion at the dose of 50 $\mu \mathrm{M}$ (Figure $1 \mathrm{~B}$ ). These results show that zerumbone suppressed BC cell proliferation, migration, and invasion in vitro.
A

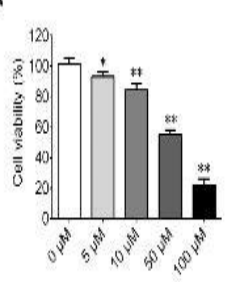

B

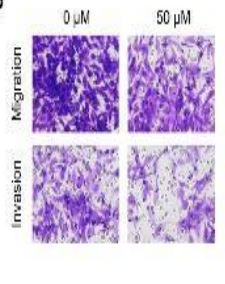

Zerumbone upregulates downregulates $\mathrm{BACH} 1$

MDA-MB-231 cells were treated with varying doses of zerumbone, and miR-708 and BACH1 expression were measured via qPCR. The results demonstrated that miR-708 and $\mathrm{BACH} 1$ were up- and downregulated, respectively (Figure $2 \mathrm{~A}$ and $\mathrm{B}$ ). Immunoblotting showed that $\mathrm{BACH} 1$ protein levels were decreased by zerumbone in a dose-dependent manner (Figure $2 \mathrm{C})$.

A

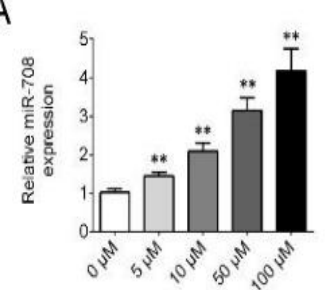

B

C

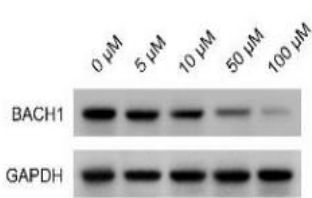

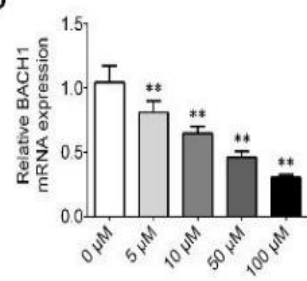

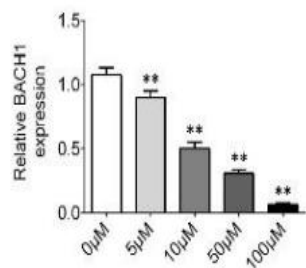

Figure 2: Zerumbone upregulates miR-708 and downregulates $\mathrm{BACH} 1$ expression. (A) Increased miR708 was observed in qPCR assays of MDA-MB-231 cells following zerumbone treatment. (B) Decreased $\mathrm{BACH} 1$ expression was observed in qPCR assays following zerumbone treatment. (C) $\mathrm{BACH} 1$ levels were confirmed by immunoblot assays; ${ }^{* *} p<0.01$ versus control

\section{Zerumbone downregulates BACH1 by upregulating miR-708}

To investigate the possible link between $\mathrm{BACH} 1$ and miR-708, a search was performed in the starBase website (http://starbase.sysu.edu.cn/ index.php) to identify potential targets of miR708. BACH1 was listed as a putative target of miR-708 (Figure $3 \mathrm{~A}$ ). Luciferase assays were performed to validate the interaction between $\mathrm{BACH} 1$ and miR-708. Wild-type BACH1 luciferase activity was significantly decreased in miR-708 mimic-transfected cells (Figure $3 \mathrm{~B}$ ). However, mutant BACH1 luciferase activity was not significantly different in $\mathrm{BC}$ cells transfected with miR-708 mimic, indicating that miR-708 interacted with $\mathrm{BACH} 1$. Moreover, zerumbone treatment decreased $\mathrm{BACH} 1$ levels, which were increased after addition of an miR-708 inhibitor (Figure $3 \mathrm{C}$ ). 
A
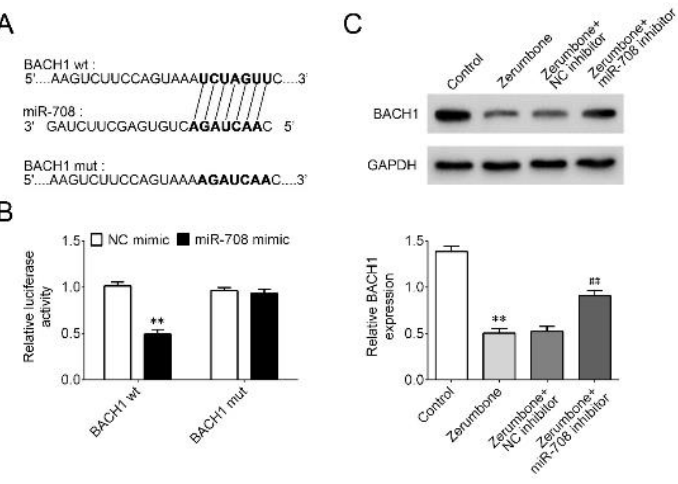

Figure 3: Zerumbone downregulates $\mathrm{BACH} 1$ via upregulating miR-708. (A) The predicted binding sites between $\mathrm{BACH} 1$ and miR-708 and the BACH1 mutant sequence. (B) Luciferase assays for $\mathrm{BACH} 1(\mathrm{Wt})$ or BACH1 (Mut) reporter activity following miR-708 mimic transfection in MDA-MB-231 cells. (C) Immunoblotting showing that the zerumbone-induced reduction of $\mathrm{BACH} 1$ could be reversed by miR-708 inhibitor; ${ }^{* *} p<0.01$ and ${ }^{\# \#} p<0.01$ versus control

Zerumbone inhibits BC cell proliferation, migration, and invasion by suppressing BACH1 expression

The final set of experiments examined whether zerumbone affected BC cell proliferation, migration, and invasion through $\mathrm{BACH} 1$. Cell proliferation was assessed in zerumbone-treated $\mathrm{BC}$ cells with or without $\mathrm{BACH} 1$ overexpression. The results revealed that $\mathrm{BACH} 1$ transfection blocked the anti-proliferative effect of zerumbone (Figure $4 \mathrm{~A}$ ). Consistent with this observation, $\mathrm{BACH} 1$ also reversed the inhibitory effects of zerumbone on $\mathrm{BC}$ cell migration and invasion as indicated by MMP2 and -9 levels (Figure $4 \mathrm{~B}$ ). Taken together, these findings support the hypothesis that zerumbone inhibits BC cell proliferation, invasion, and migration by regulating $\mathrm{BACH} 1$.

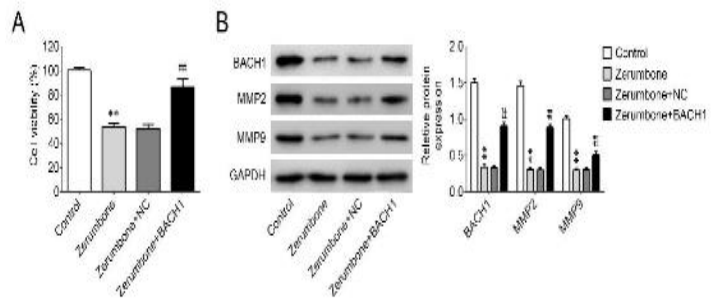

Figure 4: Zerumbone downregulates $\mathrm{BACH} 1$ by upregulating miR-708. (A) Cell viability assays showing that zerumbone's inhibition of cell proliferation was abrogated by $\mathrm{BACH} 1$ overexpression. (B) Transwell assays showing that decreased invasion in the presence of zerumbone was reversed by $\mathrm{BACH} 1$ overexpression; ${ }^{* *} p<0.01,{ }^{\# \#} p<0.01$, versus control group

\section{DISCUSSION}

In recent years, several subsets of $B C$ have been described, and the associated morbidity and mortality are high [15]. Beyond surgical resection and chemoradiotherapy, a variety of new treatment methods have helped improve BC patient prognosis and the cure rate of advanced patients [16]. Endocrine and targeted therapies for BC have made progress, and traditional Chinese medicine also has unique advantages in the fight against advanced BC [17]. A variety of traditional Chinese medicine extracts, such as the active triterpenoid monomer compound KHF16, have significant anti-tumor effects and inhibit BC cell proliferation $[18,19]$. To further improve the prognosis of patients with advanced $B C$, it is necessary to identify effective anti-tumor Chinese medicine extracts and characterize their effects.

MTT viability and colony formation assay data revealed that zerumbone suppressed $\mathrm{BC}$ cell proliferation in a concentration-dependent manner. Wound closure and Transwell assays further demonstrated that zerumbone has inhibitory effects on BC cell migration and invasion. Immunoblotting, qPCR, and luciferase assays further confirmed the compound's antitumor effects on BC through the miR-708$\mathrm{BACH} 1$ axis. This is in line with previous publications describing the anti-tumor effects of zerumbone [20].

One group reported that zerumbone inhibited colorectal cancer cell invasion via the FAk/PI3K/NFKB-uPA pathway [9]. Another found that zerumbone suppressed esophageal cancer cell migration by promoting Rac1 ubiquitination [21]. Zerumbone can affect the proliferation, migration, invasion, and apoptosis of multiple types of cancers, such as prostate, cervical, hepatoma, and oral cancers [22]. These studies, together with our findings, demonstrate that zerumbone exerts anti-tumor effects through different pathways.

Zerumbone affects multiple cellular processes by targeting miRNAs [12]. Zerumbone suppressed epithelial-mesenchymal transition by inhibiting beta-catenin signaling through regulation of miR200c [12]. Another study demonstrated that zerumbone ameliorated high-fat diet-induced adiposity via targeting miR-146b [13]. Interestingly, miR-708 was down-regulated in BC tissues, and a previous study found that miR-708 upregulation was correlated with tumor metastasis [23]. In the present study, we observed that zerumbone regulated miR-708 expression and might suppress BC progression. 
The results reveal a novel mechanism underlying the anti-tumor activity of zerumbone.

$\mathrm{BACH} 1$ is an oncogene that affects the progression and metastasis of multiple types of tumors, including pancreatic, lung, and colorectal cancers [24]. BACH1 serves as a prognostic biomarker and affects $\mathrm{BC}$ cell migration and apoptosis [25]. Collectively, our results show that zerumbone affected $\mathrm{BC}$ cell proliferation, migration, and invasion by targeting miR-708 and $\mathrm{BACH} 1$ and suggest that identifying inhibitors that target $\mathrm{BACH} 1$ could be an effective therapeutic strategy for $\mathrm{BC}$.

\section{CONCLUSION}

Zerumbone suppresses the proliferation, migration, and invasion of $\mathrm{BC}$ cells in vitro. These effects are due to zerumbone's ability to upregulate miR-708 and suppress BACH1 expression. Thus, zerumbone is a promising drug for the management of $\mathrm{BC}$.

\section{DECLARATIONS}

\section{Conflict of interest}

No conflict of interest is associated with this work.

\section{Contribution of authors}

We declare that this work was done by the authors named in this article and all liabilities pertaining to claims relating to the content of this article will be borne by the authors. Fuguang Zhao and Yong Cai designed the study, supervised the data collection, and analyzed the data; Bo Ma and Zhenye Lv interpreted the data and prepared the manuscript for publication; Jie Chen, Yuanjie Cai, and Chunjing Xu supervised the data collection, analyzed the data, and reviewed the draft of the manuscript. All authors read and approved the manuscript.

\section{Open Access}

This is an Open Access article that uses a funding model which does not charge readers or their institutions for access and distributed under the terms of the Creative Commons Attribution License (http://creativecommons.org/licenses/by/ 4.0) and the Budapest Open Access Initiative (http://www.budapestopenaccessinitiative.org/rea d), which permit unrestricted use, distribution, and reproduction in any medium, provided the original work is properly credited.

\section{REFERENCES}

1. Lopez-Rivera $V$, Zhu $P$, Dono A, Lee $S$, Chen $P R$, Ballester L, Sheth SA, Esquenazi Y. Increased Risk of Subsequent Meningioma Among Women with Malignant Breast Cancer. World Neurosurg 2020. DOI : 10.1016/j.wneu.2020.03.203

2. Bodoor K, Abu-Sheikha A, Matalka I, Alzou'bi H, Batiha O, Abu-Awad A, Jalboush S, Fayyad L, Qadiri E, Jarun $Y$. Immunohistochemical analysis of heat shock proteins in triple negative breast cancer: HSP60 expression is a marker of poor prognosis. Eur J Gynaecol Oncol 2018; 39(6): 926-934.

3. Malagobadan S, Ho CS, Nagoor NH. MicroRNA-6744-5p promotes anoikis in breast cancer and directly targets NAT1 enzyme. Cancer Biol Med 2020; 17(1): 101-111.

4. Gong Z, Chen $M$, Ren $Q$, Yue X, Dai Z. Fibronectintargeted dual-acting micelles for combination therapy of metastatic breast cancer. Signal Transduct Target Ther 2020; 5(1): 12.

5. Fisher MF, Rao SS. Three dimensional culture models to study drug resistance in breast cancer. Biotechnol Bioeng 2020; 117: 2262-2278.

6. Szabolcs A, Tiszlavicz L, Kaszaki J, Posa A, Berko A, Varga IS, Boros I, Szuts V, Lonovics J, Takacs $T$. Zerumbone exerts a beneficial effect on inflammatory parameters of cholecystokinin octapeptide-induced experimental pancreatitis but fails to improve histology. Pancreas 2007; 35(3): 249-255.

7. Girisa S, Shabnam B, Monisha J, Fan L, Halim CE, Arfuso F, Ahn KS, Sethi G, Kunnumakkara AB. Potential of Zerumbone as an Anti-Cancer Agent. Molecules 2019; 24(4): 734.

8. Jegannathan $S D$, Arul S, Dayalan $H$. Zerumbone, a Sesquiterpene, Controls Proliferation and Induces Cell Cycle Arrest in Human Laryngeal Carcinoma Cell Line Hep-2. Nutr Cancer 2016; 68(5): 865-872.

9. Kang CG, Lee HJ, Kim SH, Lee EO. Zerumbone Suppresses Osteopontin-Induced Cell Invasion Through Inhibiting the FAK/AKT/ROCK Pathway in Human NonSmall Cell Lung Cancer A549 Cells. J Nat Prod 2016; 79(1): 156-160.

10. Hosseini N, Khoshnazar A, Saidijam M, Azizi Jalilian F, Najafi R, Mahdavinezhad A, Ezati R, Sotanian A, Amini $R$. Zerumbone Suppresses Human Colorectal Cancer Invasion and Metastasis via Modulation of FAk/PI3k/NFkappaB-uPA Pathway. Nutr Cancer 2019; 71(1): 159-171.

11. E EME, A SA, Koosha S, A AA, Azam F, I MT, Khalilullah $H$, Sadiq Al-Qubaisi $M, M A A$. Zerumbone Induces Apoptosis in Breast Cancer Cells by Targeting alphavbeta3 Integrin upon Co-Administration with TP5iRGD Peptide. Molecules 2019; 24(14).

12. Dermani FK, Amini R, Saidijam M, Pourjafar M, Saki S, Najafi $R$. Zerumbone inhibits epithelial-mesenchymal transition and cancer stem cells properties by inhibiting the beta-catenin pathway through miR-200c. J Cell Physiol 2018; 233(12): 9538-9547.

Trop J Pharm Res, July 2020; 19(7): 1415 
13. Ahn J, Lee H, Jung $\mathrm{CH}$, Choi WH, Ha TY. Zerumbone ameliorates high-fat diet-induced adiposity by restoring AMPK-regulated lipogenesis and microRNA146b/SIRT1-mediated adipogenesis. Oncotarget 2017; 8(23): 36984-36995.

14. Rajan I, Jayasree PR, Kumar PR. Zerumbone induces mitochondria-mediated apoptosis via increased calcium, generation of reactive oxygen species and upregulation of soluble histone $\mathrm{H} 2 \mathrm{AX}$ in $\mathrm{K} 562$ chronic myelogenous leukemia cells. Tumour Biol 2015; 36(11): 8479-8489.

15. Ding Y, Ding K, Qian H, Yu X, Zou D, Yang H, Mo W, He $X$, Zhang $F$, Qin $C$ et al. Impact on survival of estrogen receptor, progesterone receptor and Ki-67 expression discordance pre- and post-neoadjuvant chemotherapy in breast cancer. PLoS One 2020; 15(4): e0231895.

16. Choi C, Cho WK, Park S, Shin SW, Park W, Kim H, Choi $D H$. Checkpoint Kinase 1 (CHK1) Inhibition Enhances the Sensitivity of Triple-Negative Breast Cancer Cells to Proton Irradiation via Rad51 Downregulation. Int J Mol Sci 2020; 21(8). DOI : 10.3390/ijms21082691.

17. Purrington KS, Knight J, 3rd, Dyson G, Ali-Fehmi R, Schwartz AG, Boerner JL, Bandyopadhyay S. CLCA2 expression is associated with survival among African American women with triple negative breast cancer. PLoS One 2020; 15(4): e0231712.

18. Kong Y, Li F, Nian Y, Zhou Z, Yang R, Qiu MH, Chen C. KHF16 is a Leading Structure from Cimicifuga foetida that Suppresses Breast Cancer Partially by Inhibiting the NF-kappaB Signaling Pathway. Theranostics 2016; 6(6): 875-886.

19. Hao MH, Zhang F, Liu XX, Zhang F, Wang LJ, XU SJ, Zhang $\mathrm{JH}, \mathrm{Ji} H \mathrm{HL}, \mathrm{X} U \mathrm{P}$. Qualitative and quantitative analysis of catechin and quercetin in flavonoids extracted from Rosa roxburghii Tratt. Trop J Pharm Res 2018; 17(1): 71-76.

20. Chan ML, Liang JW, Hsu LC, Chang WL, Lee SS, Guh $\mathrm{JH}$. Zerumbone, a ginger sesquiterpene, induces apoptosis and autophagy in human hormone-refractory prostate cancers through tubulin binding and crosstalk between endoplasmic reticulum stress and mitochondrial insult. Naunyn Schmiedebergs Arch Pharmacol 2015; 388(11): 1223-1236.

21. Wang M, Niu J, Gao L, Gao Y, Gao S. Zerumbone inhibits migration in ESCC via promoting Rac1 ubiquitination. Biomed Pharmacother 2019; 109(24472455.

22. LV T, Zhang $W$, Han X. Zerumbone suppresses the potential of growth and metastasis in hepatoma HepG2 cells via the MAPK signaling pathway. Oncol Lett 2018; 15(5): 7603-7610.

23. Takada Y, Murakami A, Aggarwal BB. Zerumbone abolishes NF-kappaB and IkappaBalpha kinase activation leading to suppression of antiapoptotic and metastatic gene expression, upregulation of apoptosis, and downregulation of invasion. Oncogene 2005; 24(46): 6957-6969.

24. Ou X, Gao G, Bazhabayi M, Zhang K, Liu F, Xiao X. MALAT1 and BACH1 are prognostic biomarkers for triple-negative breast cancer. J Cancer Res Ther 2019; 15(7): 1597-1602.

25. De Nicolo A, Tancredi M, Lombardi G, Flemma CC, Barbuti S, Di Cristofano C, Sobhian B, Bevilacqua G, Drapkin R, Caligo MA. A novel breast cancer-associated BRIP1 (FANCJ/BACH1) germ-line mutation impairs protein stability and function. Clin Cancer Res 2008; 14(14): 4672-4680. 